\title{
Exploring Protein Stability by Comparative Molecular Dynamics Simulations of Homologous Hyperthermophilic, Mesophilic, and Psychrophilic Proteins
}

\author{
Sara Khan ${ }^{\dagger}$, Umar Farooq ${ }^{\dagger, *}$, Maria Kurnikova
}

${ }^{\dagger}$ Department of Chemistry, COMSATS Institute of Information Technology, Abbottabad 22060, Pakistan

${ }^{\ddagger}$ Department of Chemistry, Carnegie Mellon University, Pittsburgh 15213, Pennsylvania, United States

*Corresponding Author

Phone: +92-992-383591-5. E-mail: umarf@ciit.net.pk 


\section{G, P, S, T H, K, R F, W, Y I, L, M, V}

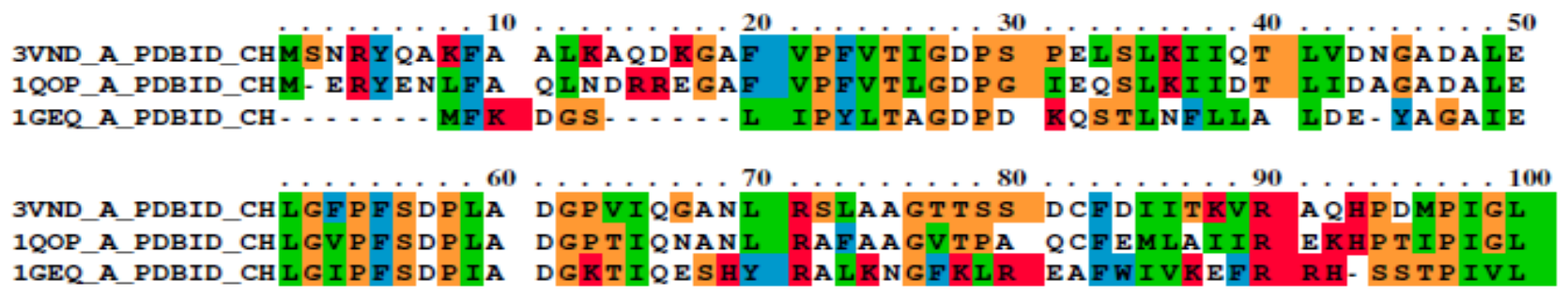

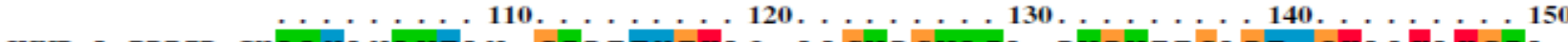
3VND_A_PDBID_CHLLYANLVFAN GIDEFYTKAQ AAGVDSVLIA DVPVEESAPF SKAAKAHGIA 1QOP_A_PDBID_CHLMYANLVFNN GIDAFYARCE QVGVDSVLVA DVPVEESAPF RQAALRHIA 1GEQ_A_PDBID_CHMTYYNPIYRA GVRNFLAEAK ASGVDGILVV DLPVFHAKEF TEIAREEGIK

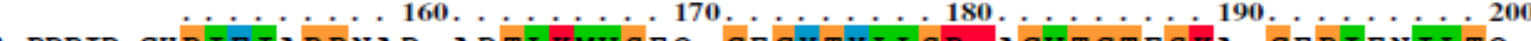
3VND_A_PDBID_CHPIFIAPPNAD ADTLKMVSEQ GEGYTYLLSR AGVTGTESKA GEPIENILTQ 10OP_A_PDBID_CHPIFICPPNAD DDLLRQVASY GRGYTYLLSR SGVTGAENRG ALPLHHLIEK 1GEQ_A_PDBD_CHTVFLAAPNTP DERLKVIDDM TTGFVYLVSL YGTTGAREEI PKTAYDLLRR

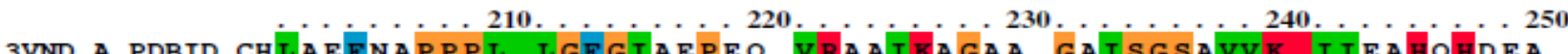
10OP_A_PDBD_CHLKEYHAAPAL QGFGISSPEQ VSAAVRAGAA GAISGSAIVK IIEKNIASPK 1GEQ_A_PDBID_CHAKRICRNKVA VGFGVSKREH VVSLLKEGAN GVVVGSALVK IIGEK-.-GR

3VND_A_PDBID_CHTLLAKLAEFT TAMKA TT.

1QOP_A_PDBID_CHQMLAELRSFV SAMKAASRA

1GEQ_A_PDBID_CHEATEFLKKKV EELLGI...

Figure S1. Residue based Alignment scheme using ClustalW color scheme $e^{1,2}$

Table S1. Cosine content on the principal component (PC1 and PC2)

\begin{tabular}{|l|l|l|l|l|l|l|l|l|}
\hline Temperature & \multicolumn{2}{|l|}{ Cosine content-250K } & \multicolumn{2}{l|}{ Cosine content-300K } & \multicolumn{2}{l|}{ Cosine content-350K } & \multicolumn{2}{l|}{ Cosine content-375K } \\
\hline Protein (TRPS) & PC1 & PC2 & PC1 & PC2 & PC1 & PC2 & PC1 & PC2 \\
\hline \multirow{3}{*}{ Psychrophilic } & $0.070^{\mathrm{a}}$ & $0.012^{\mathrm{a}}$ & $0.066^{\mathrm{a}}$ & $0.001^{\mathrm{a}}$ & $0.292^{\mathrm{a}}$ & $0.141^{\mathrm{a}}$ & $0.372^{\mathrm{a}}$ & $0.292^{\mathrm{a}}$ \\
\cline { 2 - 9 } & $0.121^{\mathrm{b}}$ & $0.09^{\mathrm{b}}$ & $0.121^{\mathrm{b}}$ & $0.093^{\mathrm{b}}$ & $0.214^{\mathrm{b}}$ & $0.103^{\mathrm{b}}$ & $0.312^{\mathrm{b}}$ & $0.195^{\mathrm{b}}$ \\
\hline \multirow{3}{*}{ Mesophilic } & $0.071^{\mathrm{a}}$ & $0.050^{\mathrm{a}}$ & $0.127^{\mathrm{a}}$ & $0.091^{\mathrm{a}}$ & $0.131^{\mathrm{a}}$ & $0.251^{\mathrm{a}}$ & $0.217^{\mathrm{a}}$ & $0.386^{\mathrm{a}}$ \\
\cline { 2 - 9 } & $0.066^{\mathrm{b}}$ & $0.113^{\mathrm{b}}$ & $0.049^{\mathrm{b}}$ & $0.113^{\mathrm{b}}$ & $0.093^{\mathrm{b}}$ & 0.312 & $0.423^{\mathrm{b}}$ & $0.198^{\mathrm{b}}$ \\
\hline \multirow{2}{*}{ Hyperthermophilic } & $0.039^{\mathrm{a}}$ & $0.024^{\mathrm{a}}$ & $0.036^{\mathrm{a}}$ & $0.004^{\mathrm{a}}$ & $0.236^{\mathrm{a}}$ & $0.014^{\mathrm{a}}$ & $0.115^{\mathrm{a}}$ & $0.620^{\mathrm{a}}$ \\
\cline { 2 - 9 } & $0.025^{\mathrm{b}}$ & $0.052^{\mathrm{b}}$ & $0.049^{\mathrm{b}}$ & $0.101^{\mathrm{b}}$ & $0.302^{\mathrm{b}}$ & $0.567^{\mathrm{b}}$ & $0.342^{\mathrm{b}}$ & $0.012^{\mathrm{b}}$ \\
\hline
\end{tabular}

${ }^{\mathrm{a}}$ Attributes to cosine content from PC computed for $\mathrm{C} \alpha$ atoms for $0-15 \mathrm{~ns}$. ${ }^{\mathrm{b}}$ Attributes to cosine content from PC computed for $\mathrm{C} \alpha$ atoms for $16-30 \mathrm{~ns}$ 


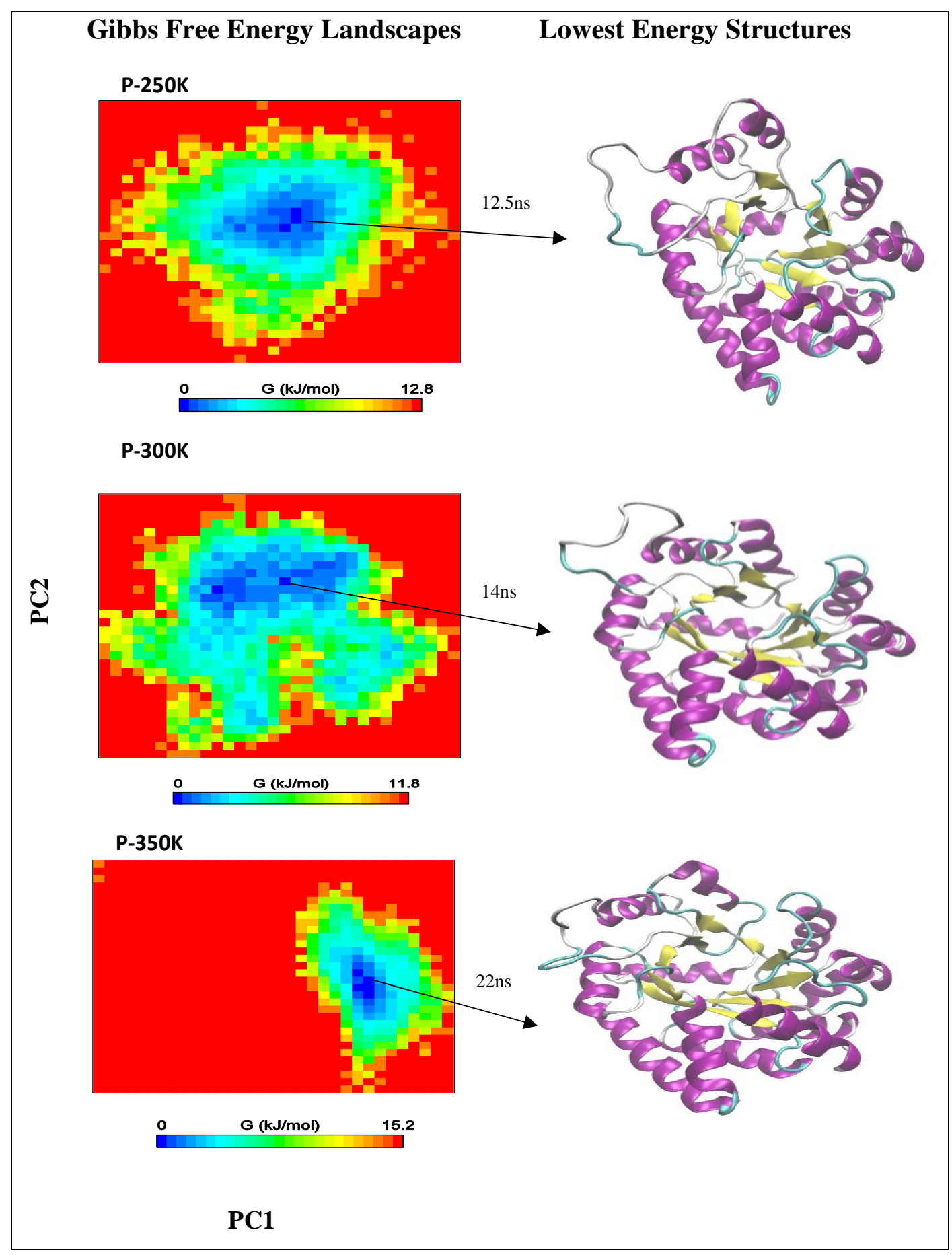




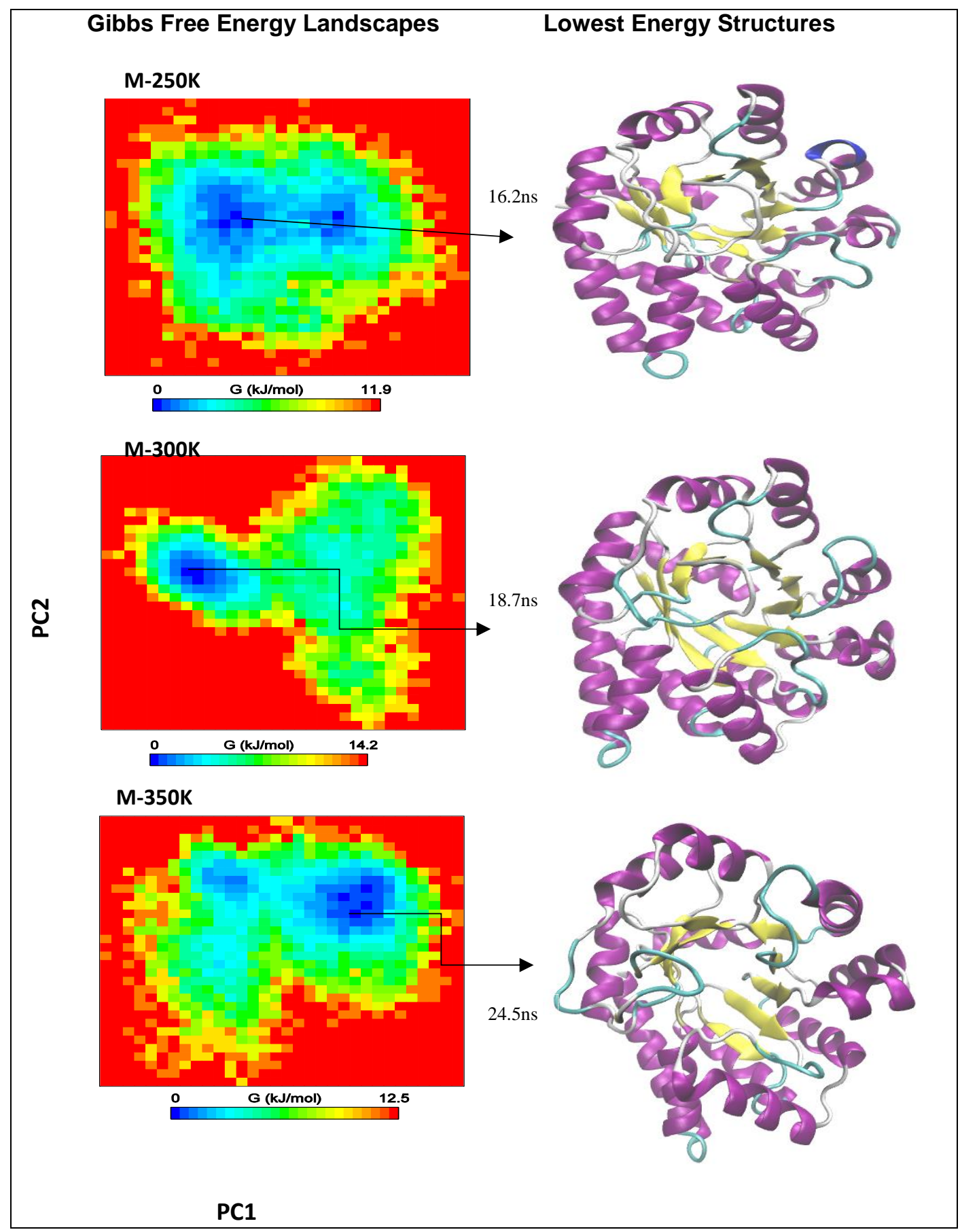




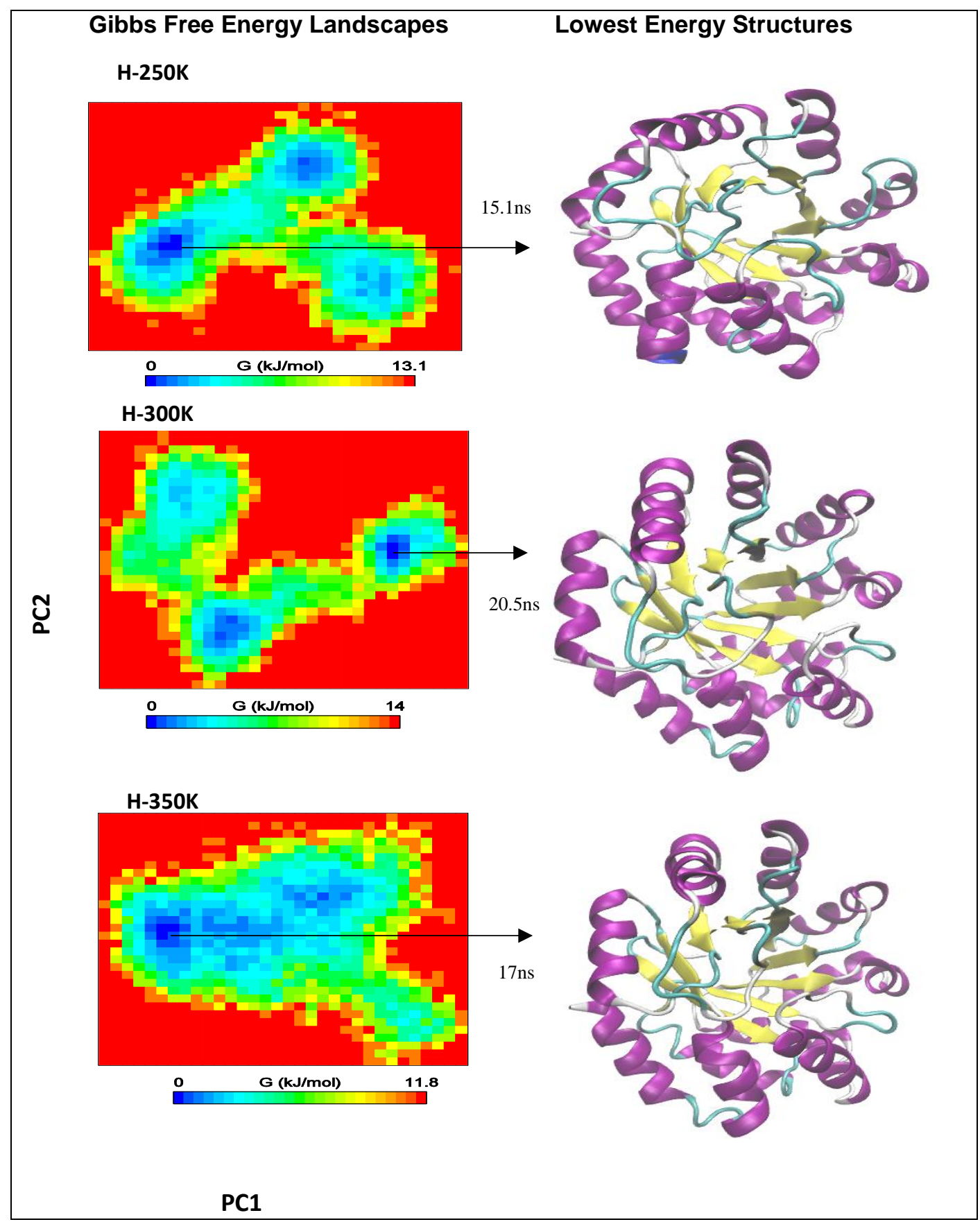

Figure S2. Free energy landscapes (FEL) and corresponding representative structures from the MD trajectories for psychrophilic (P), mesophilic (M) and hyperthermophilic (H) TRPS at 250, 300, and $350 \mathrm{~K}$. The FELs were obtained using as reaction coordinates the projections of TRPS $\mathrm{C} \alpha$ atoms onto the first two principal components. Free energy values are given in $\mathrm{kcal} \mathrm{mol}^{-1}$ as indicated by the color bars. 
Table S2. Salt bridges with life span $>70 \%$ of the simulated time at 250, 300, 350, and $375 \mathrm{~K}$ in psychrophilic (a), mesophilic (b) and hyperthermophilic (c) TRPS ( $\alpha$-subunit) proteins are given, color codes represent Intrahelix, loop-helix sheet-helix, interhelix, sheet-loop. (|) represent chains of salt bridges, while salt bridges extended to neighboring residues are placed in single box.

\begin{tabular}{|c|c|c|c|}
\hline \multicolumn{4}{|c|}{ Table S2 (a). Psychrophilic TRPS ( $\alpha$-subunit) Avg. Life span >70\% } \\
\hline 250 K & $300 \mathrm{~K}$ & $350 \mathrm{~K}$ & $375 \mathrm{~K}$ \\
\hline E 219-R222 & E 219-R222 & E 219-R222 & - \\
\hline P125-R4 & P 125-R4 & D 125-R4 & P 125-R4 \\
R90 \\
R90
\end{tabular}

\begin{tabular}{|c|c|c|c|}
\hline \multicolumn{4}{|c|}{ Table S2 (b). Mesophilic TRPS ( $\alpha$-subunit) Avg. Life span >70\% } \\
\hline 250 K & $300 \mathrm{~K}$ & $350 \mathrm{~K}$ & $375 \mathrm{~K}$ \\
\hline P123-R88 & D123-R88 & D123-R88 & D123-R88 \\
R2 R2 & D26-R69 & D26-R69 \\
\hline D26-R69 & D26-R69 & & E241 \\
E241 & & D41-R255 & - \\
\hline- & D41-R255 & E15-R266 & - \\
\hline E15-R266 & E15-R266 & R262 & - \\
\hline E241-K238 & R262 & E241-K238 & D37-K90 \\
\hline E30-K34 & E30-K34 & D37-K90 & E4-R170 \\
\hline D37-K90 & D37-K90 & & - \\
\hline E4-R170 & E4-R170 & & - \\
\hline
\end{tabular}




\begin{tabular}{|c|c|c|c|}
\hline \multicolumn{2}{|c|}{ Table S2 (c). Hyperthermophilic TRPS ( $\alpha$-subunit) Avg. Life span >70\% } \\
\hline 250 K & $300 \mathrm{~K}$ & $350 \mathrm{~K}$ & $375 \mathrm{~K}$ \\
\hline D17-R232 & D17-R232 & D17-R232 & K18-E67 \\
D15-R57 & D15-R57 & D15-R57 & D17-R232 \\
& & & D15-R57 \\
\hline D181-R184 & D181-R184 & D181-R184 & E212-R184 \\
E212 & E212 & E212 & \\
\hline E127-R130 & E127-R130 & E127-R130 & E127-R130 \\
\hline D153-R191 & - & D153-R191 & D153-R191 \\
\hline D4-K193 & D4-K193 & & D4-K193 \\
\hline D110-R76 & D110-R76 & D110-R76 & D110-R76 \\
E74-R77 & E74-R77 & E74-R77 & E74-R77 \\
\hline E244-K241 & E244-K241 & E244-K241 & E244-K241 \\
\hline D146-R185 & D146-R185 & D146-R185 & D146-R185 \\
& E147-K150 & E147-K150 & E147-K150 \\
\hline
\end{tabular}
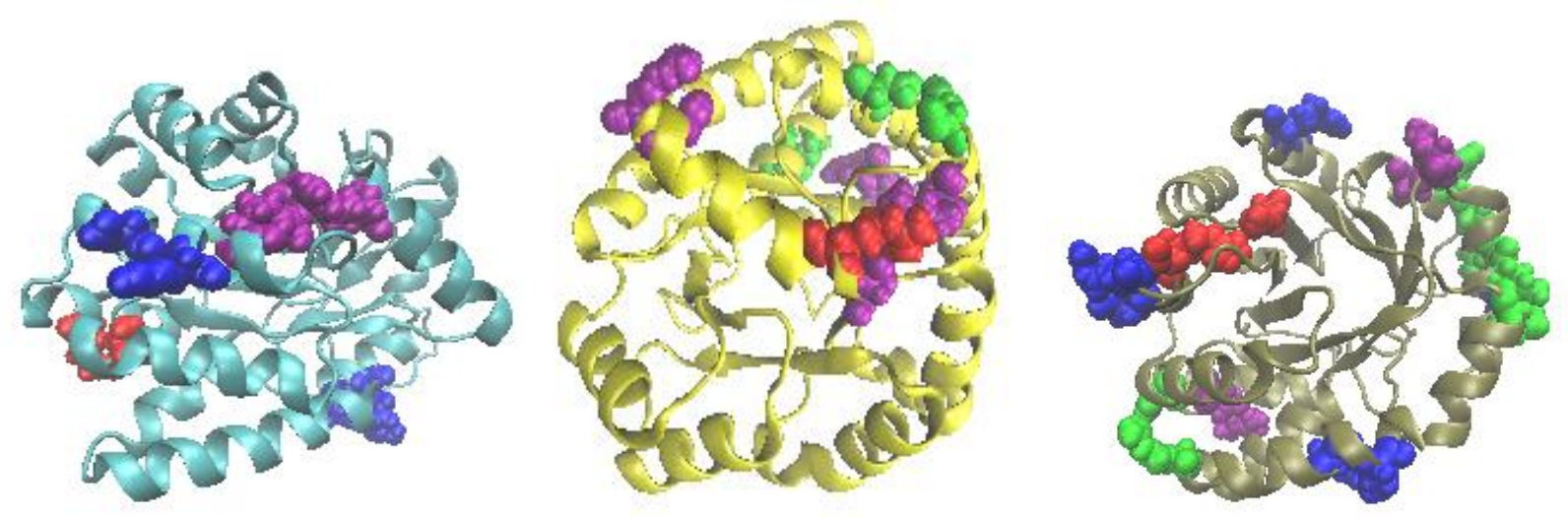

Figure S3. Positions of the salt bridges with highest life span. Psychrophilic (light blue), mesophilic (yellow) and hyperthermophilic (tan) TRPS $\alpha$-subunit with salt bridges having life span $>70 \%$ present at all temperatures simulations.

\section{References:}

1. Larkin, M. A.; Blackshields, G.; Brown, N.; Chenna, R.; McGettigan, P. A.; McWilliam, H.; Valentin, F.; Wallace, I. M.; Wilm, A.; Lopez, R., Clustal W and Clustal X version 2.0. Bioinformatics 2007, 23, 2947-2948 10.1093/bioinformatics/btm404.

2. Thompson, J. D.; Gibson, T. J.; Plewniak, F.; Jeanmougin, F.; Higgins, D. G., The CLUSTAL_X windows interface: Flexible Strategies for Multiple Sequence Alignment Aided by Quality Analysis Tools. Nucleic Acids Res. 1997, 25, 4876-4882 10.1093/nar/25.24.4876. 